\title{
MTSS1 and SCAMP1 cooperate to prevent invasion in breast cancer
}

\author{
Jayakumar Vadakekolathu', Shaymaa Ismael Kadhim Al-Juboori ${ }^{1,3}$, Catherine Johnson ${ }^{1}$, Anne Schneider ${ }^{1}$, \\ Magdalena Elżbieta Buczek', Anna Di Biase', Alan Graham Pockley (1)', Graham Roy Ball', \\ Desmond George Powe ${ }^{1,2}$ and Tarik Regad ${ }^{1}$
}

\begin{abstract}
Cell-cell adhesions constitute the structural "glue" that retains cells together and contributes to tissue organisation and physiological function. The integrity of these structures is regulated by extracellular and intracellular signals and pathways that act on the functional units of cell adhesion such as the cell adhesion molecules/adhesion receptors, the extracellular matrix (ECM) proteins and the cytoplasmic plaque/peripheral membrane proteins. In advanced cancer, these regulatory pathways are dysregulated and lead to cell-cell adhesion disassembly, increased invasion and metastasis. The Metastasis suppressor protein 1 (MTSS1) plays a key role in the maintenance of cell-cell adhesions and its loss correlates with tumour progression in a variety of cancers. However, the mechanisms that regulate its function are not well-known. Using a system biology approach, we unravelled potential interacting partners of MTSS1. We found that the secretory carrier-associated membrane protein 1 (SCAMP1), a molecule involved in post-Golgi recycling pathways and in endosome cell membrane recycling, enhances Mtss1 anti-invasive function in HER2+/ER-/PRbreast cancer, by promoting its protein trafficking leading to elevated levels of RAC1-GTP and increased cell-cell adhesions. This was clinically tested in HER2 breast cancer tissue and shown that loss of MTSS1 and SCAMP1 correlates with reduced disease-specific survival. In summary, we provide evidence of the cooperative roles of MTSS1 and SCAMP1 in preventing HER2+/ER-/PR - breast cancer invasion and we show that the loss of Mtss1 and Scamp1 results in a more aggressive cancer cell phenotype.
\end{abstract}

\section{Introduction}

Metastasis is a process by which cancer cells that acquired high migratory and invasive properties, leave primary tumours and migrate through the vascular and lymphatic circulatory system to other tissues where they form secondary tumours ${ }^{1}$. This process requires the inactivation of cellular and molecular pathways that maintain cell-cell adhesion and regulate cytoskeleton remodelling and cell motility ${ }^{2}$. In breast cancer 1:5

Correspondence: Tarik Regad (tarik.regad@ntu.ac.uk)

${ }^{1}$ The John van Geest Cancer Research Centre, School of Science and

Technology, Nottingham Trent University, Nottingham NG11 8NS, UK

2Department of Cellular Pathology, Queen's Medical Centre, Nottingham

University Hospitals Trust, Nottingham NG7 2UH, UK

Full list of author information is available at the end of the article

These authors jointly supervised this work: Desmond George Powe and Tarik Regad.

These authors contributed equally: Jayakumar Vadakekolathu and Shaymaa Ismael Kadhim Al-Juboori. 
MTSS1 belongs to the IMD-family (IRSp53 and MIM (Missing in metastasis) domain) and serves as an actinbinding scaffold protein that is implicated in carcinogenesis and metastasis. It has been proposed that MTSS1 promotes the assembly of actin filaments, and is associated with cytoskeletal organisation and cell motility through elevating RAC1-GTP expression ${ }^{5-7}$. This effect accelerates the kinetics of adherens junction assembly and therefore cell-cell adhesions ${ }^{7}$. MTSS1 is highly expressed in some cancer types and its loss correlates with metastasis and poor prognosis, including breast cancer ${ }^{8,9}$. However, the mechanisms and molecular pathways that regulate the function of MTSS1 are less known. Analysis of the MTSS1 hub of connectivity unravelled several potential interacting partners including the secretory carrier-associated membrane protein 1 (SCAMP1). This molecule belongs to a family of membrane proteins that are involved in postGolgi recycling pathways and endosome cell membrane recycling ${ }^{10,11}$. The intracellular trafficking of membrane vesicles plays an essential role in the maintenance and the regulation of components of the plasma membrane. Alterations in this cellular pathway can affect cell-cell adhesions and may result in increased cell motility and invasion of cancer cells ${ }^{12}$. On the basis of this background, we hypothesised that the vesicle carrier protein SCAMP1 is involved in stabilising MTSS1 protein trafficking that promotes MTSS1 anti-invasive and anti-metastatic functions by endorsing cell-cell adhesion in HER2+ breast cancer. Moreover, we reveal the dual role of MTSS1 and SCAMP1 in preventing HER2+ breast cancer progression. To better understand the role of MTSS1 and SCAMP1 in tumour progression, we investigated their influence on cell migration and invasion using HER2+ breast cancer cell lines, and MTSS1-expressing and SCAMP1-expressing constructs. Furthermore, we determined the translational importance of this proposal in a clinical setting by showing that loss of MTSS1 and SCAMP1 expression are specifically associated with a worse prognosis in HER2+/ER $-/ P R-$ breast cancer. These studies demonstrate that MTSS1, via the carrier protein SCAMP1, prevents cell invasion by promoting cell-cell adhesion via the induction of elevated levels of RAC1-GTP. Collectively, these results support the concept that SCAMP1 promotes MTSS1 protein trafficking that potentiate anti-invasive and antimetastatic functions. SCAMP1-regulated MTSS1 prevents a more aggressive cancer cell phenotype and its loss is responsible for reduced survival in patients with HER2 +/ER-/PR- breast cancer.

\section{Results}

Identification of MTSS1 and SCAMP1 as key regulators of HER2 + cancer progression by artificial neural network (ANN)-based integrative data mining HER2+ breast cancers are among the most aggressive type of breast cancer.
The HER2 receptor is activated upon ligand binding and mediate their influence via RAS signalling pathways that are involved in the regulation of cellular processes including proliferation, survival, and migration. In HER2+ breast cancer, ERBB2 is mutated and results in aberrant activation of RAS signalling that drives HER2+ breast cancer progression. Due to the importance of this pathway, we investigated cellular pathways that may contribute to malignancy progression using a system biology approach. A stepwise ANN method was used to identify an optimised gene signature panel that is associated with the expression of $R A S$ in a HER2+ population from the Uppsala breast cancer data set (E-GEOD 20194) (Fig. 1a). The top 100 most associated genes (out of 53,000) were selected for ANN inference (ANNi) that uses a suite of ANN models to study the inter-relationship between markers in a defined set based on the weights of the neural network model ${ }^{13,14}$. Results of the full network inference with filtering to the top 100 interaction values are presented in cytoscape (Fig. $1 \mathrm{~b}$ and supplementary figure 1). The key hubs (genes with the strongest level of interaction) identified were MTSS1, KRT35, NTRK2, SLC6A2 and COL4A4 (Supplementary figure 1). A predominance of strong positive interactions are seen with fewer negative interactions. Interestingly, MTSS1 has previously been shown to play a key role in preventing invasion and metastasis in different types of cancer ${ }^{9,15-22}$, but the mechanisms that regulate its function are unknown. This bioinformatics approach allowed the unravelling of potential interacting partners that may regulate the function of MTSS1 (Fig. 1b).

Among MTSS1-predicted interacting partners, we selected two molecules for further studies: SCAMP1 and SERPIN B13. The SCAMP1 is a molecule that is involved in post-Golgi recycling pathways and in endosome cell membrane recycling ${ }^{10,11}$. During these processes, some proteins (e.g., receptors) are a target of proteolysis involving proteinases such as Cathepsins that are involved in endosomal protein catabolism. SERPIN B13 (Hurpin/ Headpin) is an intracellular inhibitor of papain-like cysteine proteases (Cathepsins) that has been shown to inhibit specifically Cathepsin $\mathrm{K}$ and $\mathrm{L}^{23,24}$. We further hypothesised that SCAMP1 may act as a vesicular carrier for MTSS1 and that SERPIN B13 may protect MTSS1 from potential proteolysis during endosome trafficking.

\section{Clinical significance of MTSS1 and SCAMP1 expression: association with poor prognosis}

To investigate whether this hypothesis is translated in the clinical setting, we verified the presence and the expression of MTSS1, SCAMP1 and SERPIN B13 in a cohort of breast cancer patients by immunohistochemistry staining, using specific antibodies to these proteins. When present, cytoplasmic staining was detected in the malignant breast tissue epithelium, with occasional 


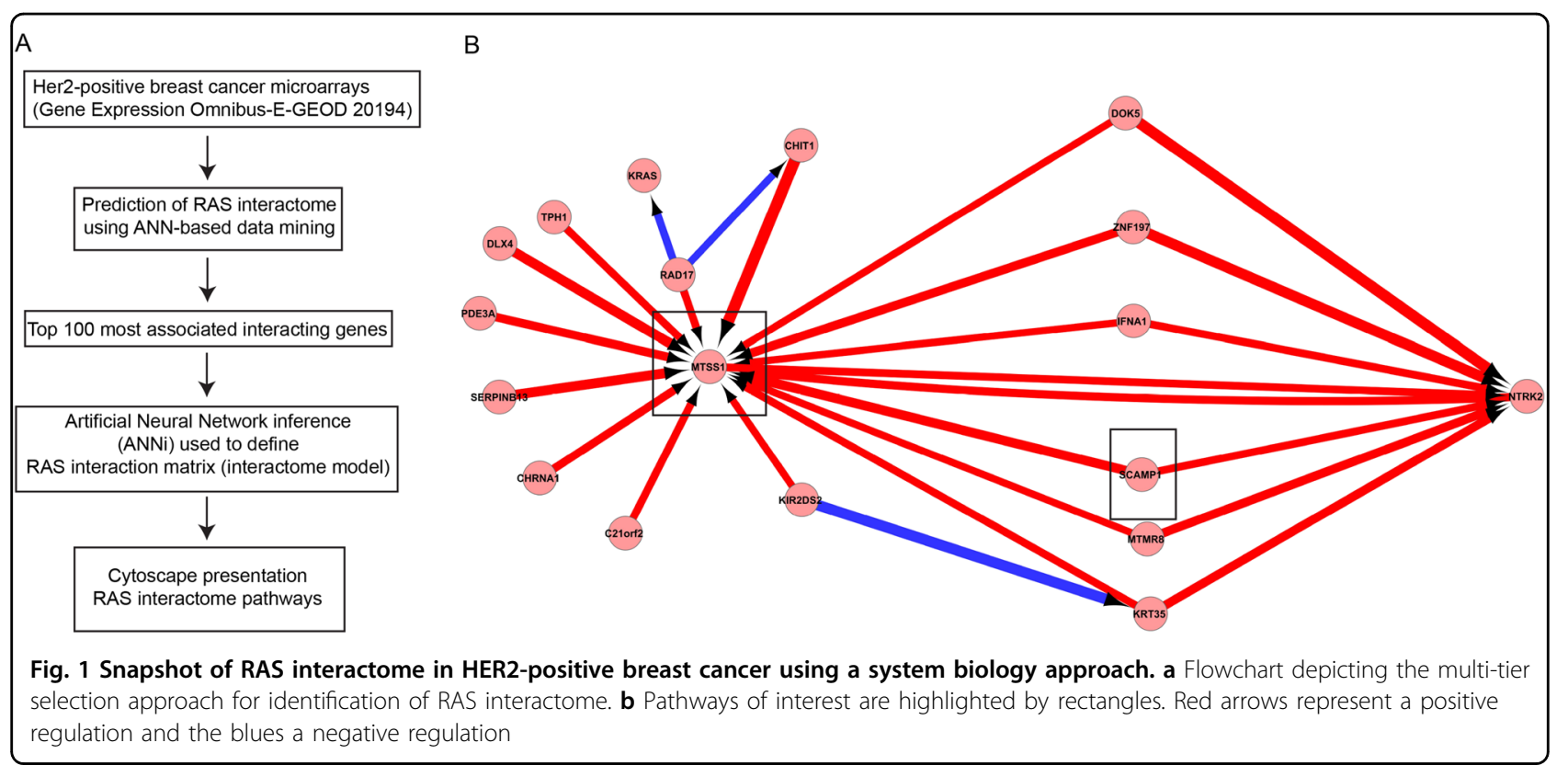

membranous-type staining seen for SCAMP1 and SERPIN B13 (Fig. 2). Expression varied according to tumour molecular phenotype with 73\%, 16 and $11 \%$ Mtss1 expression seen in oestrogen receptor (ER)-positive, ERnegative and HER $2+$ patient classes, respectively $\left(\chi^{2}=\right.$ 25.84, $p<0.0001$ ). Across the whole (unselected) population, MTSS1 expression did not associate with cancerspecific survival (Supplementary figure 2A), but a relationship was detected with MTSS1 loss and metastasis development $\left(\chi^{2}=3.83, p=0.05\right)$.

Subgroup analysis was performed to test the relationship of MTSS1 expression with disease-specific survival and metastasis outcome in HER2+ breast cancer. Expression of MTSS1 positively correlated with cancer survival $\left(x^{2}=5.81, p=0.016\right)$. Our in vitro studies demonstrated a stronger influence of MTSS1 loss with increasing tumour aggression in cancer cells with a 'pure' HER2+ phenotype (HER2+/ER-/PR-). In the clinical samples, $52 \%$ of the HER2+ patient class had the HER2 +/ER-/PR - phenotype and they differed to their hormonally (ER/PR)-positive counterparts in showing increased loss of MTSS1 ( $p=0.031$; Fig. 2g). HER2+/ER $-/ \mathrm{PR}$ - phenotype tumours showed increased loss of SCAMP1 protein compared to other HER $2+$ classes $(p=$ $0.034)$. In addition, a significant positive correlation $(r=$ $0.224, p=0.013$ ) between MTSS1 and SCAMP1 expression was identified in the 'pure' HER2 group but not in the full patient cohort (Fig. 3).

\section{Expression of MTSS1 and SCAMP1 prevent cell migration and invasion}

MTSS1 and SCAMP1 loss is associated with increased invasion that is an early event of the metastatic process and, as seen in the clinical samples, by worsened prognosis. To investigate the expression of endogenous MTSS1 and SCAMP1 in breast cancer cell lines, we performed immunoblotting (IB) using whole-cell extracts from SkBr3 and MDA-MB-453 (HER2+/ER-/PR-) and BT-474 (HER2+/ER+/PR+) human breast cancer cells. Compared to their expression in BT-474 (used as a control), SkBr3 and MDA-MB-453 expressed significantly lower levels of MTSS1 and SCAMP1 (Fig. 4a). This result was consistent with the low expression of MTSS1 and SCAMP1 that were observed in HER2+/ER - /PR - breast cancer tissues (Fig. 2). To determine whether MTSS1 and SCAMP1 interact, we performed a proximity ligation assay (PLA) and immunoprecipitation (IP) experiment using whole-cell extracts from the BT-474 breast cancer cell line. The results from these experiments demonstrated that both proteins interact (Fig. 4b, c).To investigate the role of MTSS1 and SCAMP1 in cell migration and invasion, we generated MTSS1 green fluorescent protein (GFP)-tagged and a SCAMP1 haemagglutinin (HA)-tagged constructs: MTSS1-GFP and SCAMP1-HA (Fig. 4a). The constructs were transfected in $\mathrm{SkBr} 3$ and MDA-MB-453 cell lines and their protein expression pattern was determined by IB and immunofluorescence (IF) (Fig. 4d, e). To determine the role of MTSS1 and SCAMP1 in cell migration and invasion, we used a wound-healing (Scratch assay) and well-cell invasion assays. SkBr3 and MDA-MB-453 cells expressing MTSS1 or SCAMP1 exhibited a decreased migration (Fig. 5a, b) and invasive (Fig. 5d, e) capacities in comparison to cells expressing the empty vector (Fig. 5). This effect on migration and invasion was more significant when both MTSS1 and SCAMP1 were co-expressed. An increased 


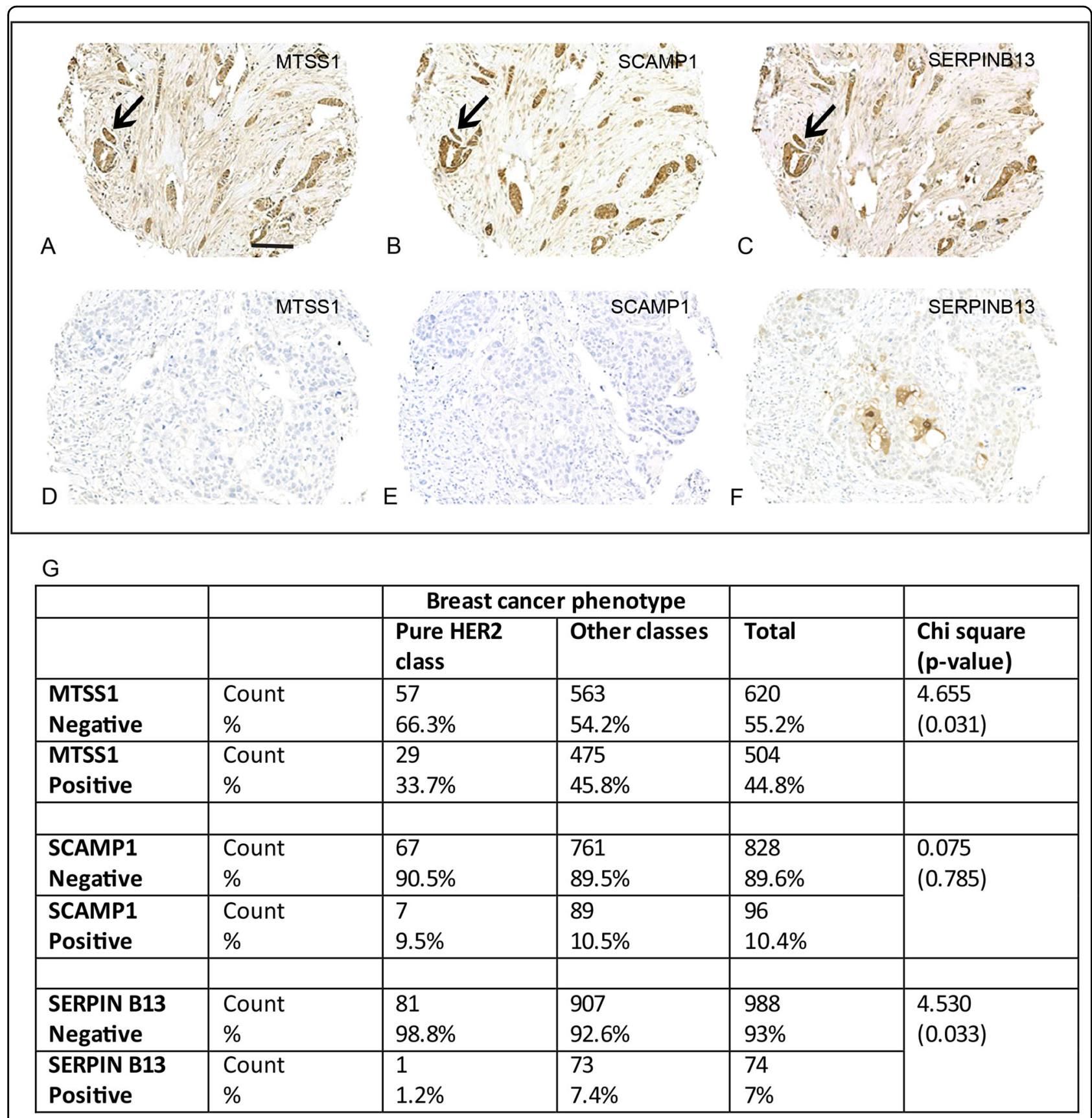

Fig. 2 Cytoplasmic staining was detected for (a) MTSS1, (b) SCAMP1 and (c) Serpin B13 protein in the malignant epithelium of breast cancer. Alternatively, the majority of 'pure' HER2+ (HER2+/ER-/PR-) tumours showed loss or in (d) MTSS1, (e) SCAMP1 and (f) SERPIN B13 staining. $\mathbf{g}$ Distribution of MTSS1, SCAMP1 and SERPIN B13 in the 'pure' HER2 patient class versus other tumour classes

cell migration of BT-474 breast cancer cells was also observed following combined MTSS1 and SCAMP1 knockdowns, and when compared to the control or when MTSS1 or SCAMP1 were individually knocked down (Fig. 5c and supplementary figure $2 \mathrm{~B}$ and $\mathrm{C}$ ). Furthermore, the expression of MTSS1, SCAMP1 or both does not appear to affect the proliferation of transfected cells (Fig. 5f, g). These results demonstrate that MTSS1 and
SCAMP1 interact and cooperate in preventing migration and invasion of HER2+/ER-/PR - breast cancer cells.

MTSS1 and SCAMP1 promote cell-cell adhesion via RAC1GTP activation in HER2 + breast cancer cell lines

The transition from non-invasive to invasive cancer is prevented by cell-cell adhesions that are maintained by collective actions of cellular pathways such as the ones 


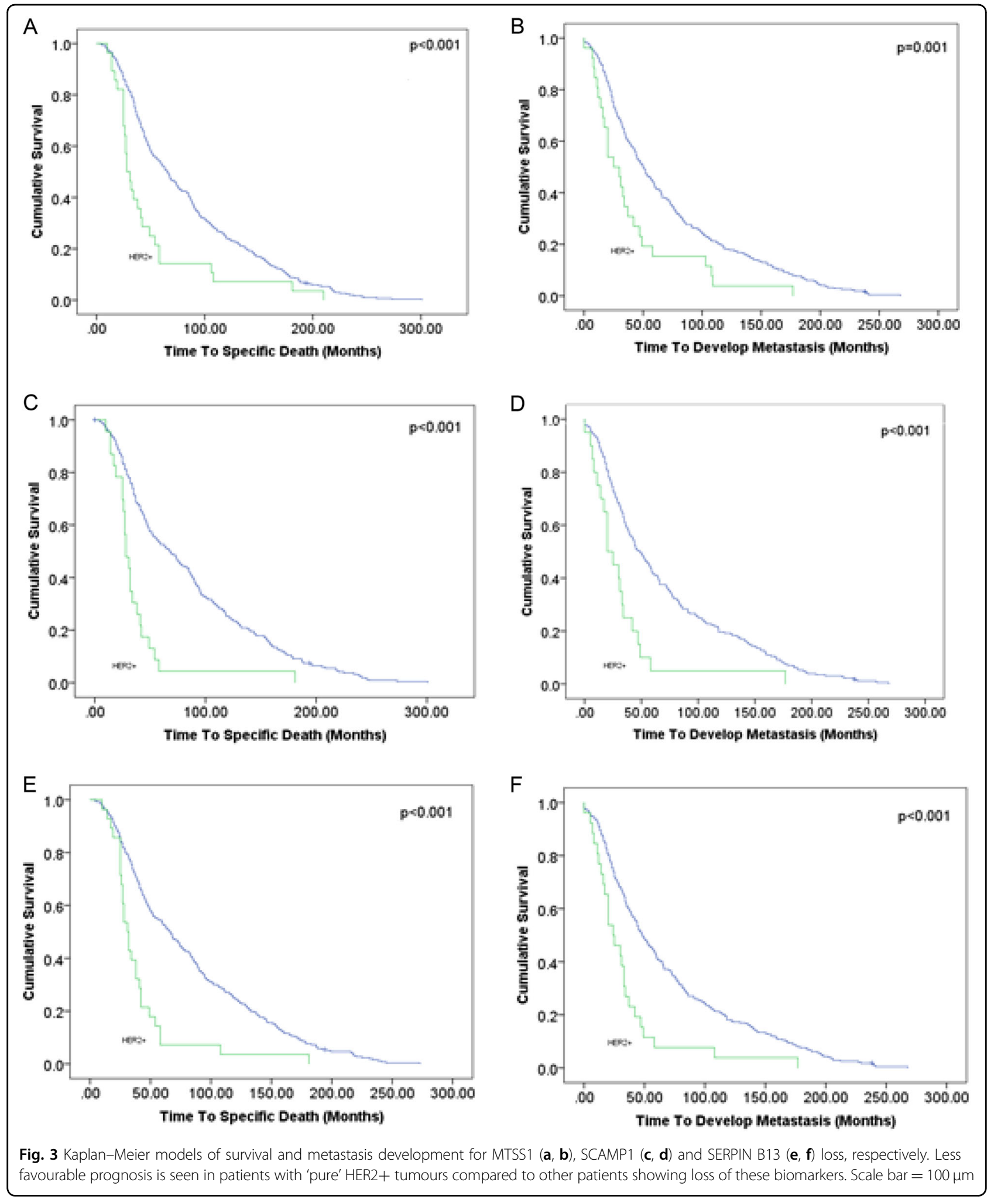

involved in the dynamic reorganisation of the actin cytoskeleton. It has previously been shown that MTSS1 accelerate the kinetics of adherens junction assembly and cell-cell adhesions through elevating RAC1-GTP expres$\operatorname{sion}^{5-7}$. To investigate the mechanism by which MTSS1 and Scamp1 prevent HER2+/ER-/PR - cell invasion, we 


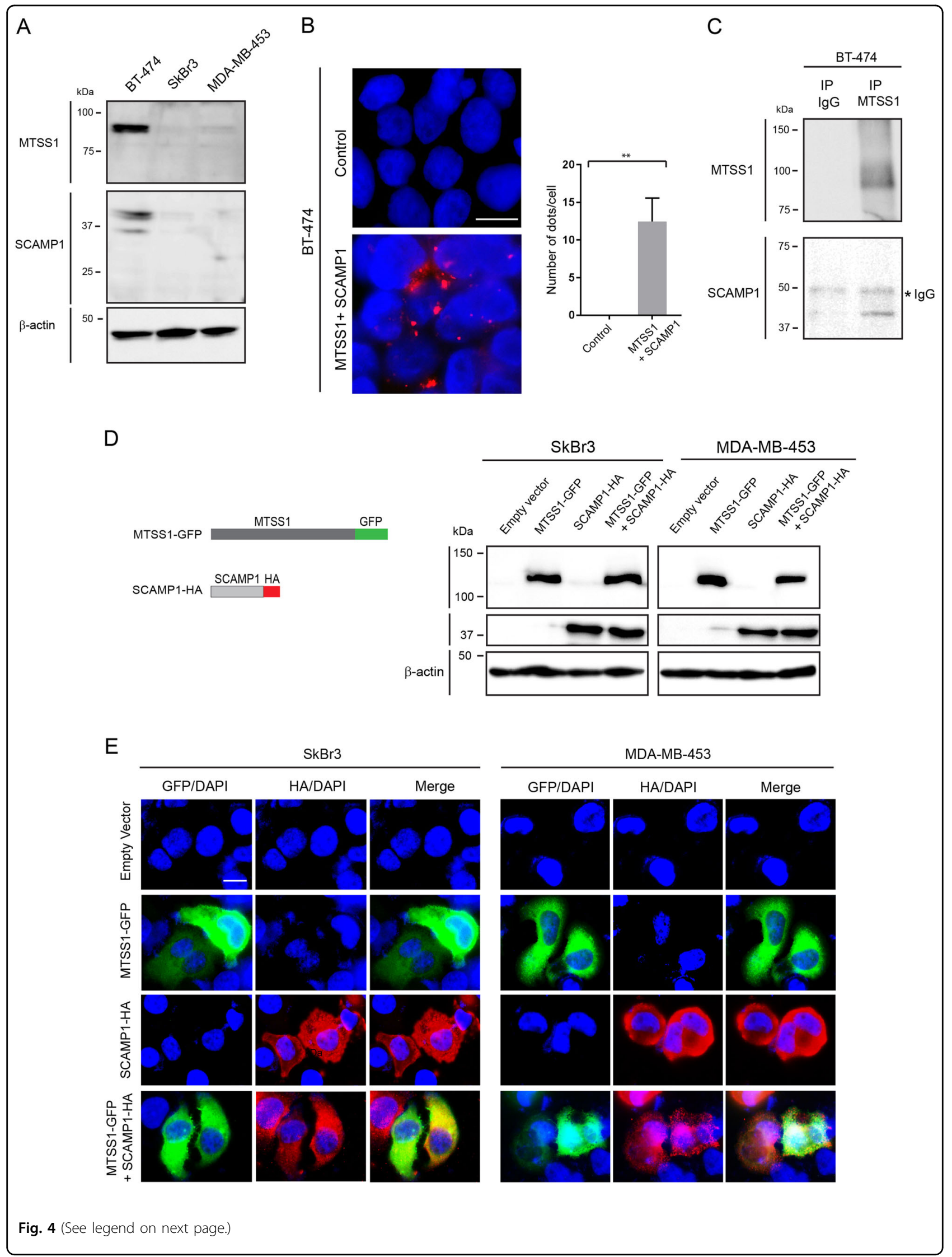


(see figure on previous page)

Fig. 4 Cellular Interactions between MTSS1 and SCAMP1 in HER2+/ER-/PR- breast cancer cell lines. a Protein expression profiles of endogenous MTSS1 and SCAMP1 as assessed by immunoblotting from BT-474, SkBr3 and MDA-MB-453 whole-cell extracts and using MTSS1 and SCAMP1 antibodies. b Proximity ligation analysis of endogenous MTSS1 and SCAMP1 interactions in BT-474 cells. Scale bar = $10 \mu \mathrm{m}$. The graph represents the number of dots/cell. Two tailed $t$-test ${ }^{* *} p=0.0024$. $\mathbf{c}$ Interaction of MTSS1 and SCAMP1 as shown by immunoblotting from anti-MTSS1 immunoprecipitates and from BT-474 whole-cell extracts using anti-MTSS1 and anti-SCAMP1 antibodies. d Schematic representation of MTSS1-GFP and SCAMP1-HA mutant constructs and their protein expression profiles as assessed by immunoblotting from SkBr3 and MDA-MB-453 whole-cell extracts and using anti-GFP and anti-HA antibodies. e Immunofluorescence images demonstrating expression of MTSS1-GFP and/or SCAMP1-HA in SkBr3 and MDA-MB-453 cells using anti-GFP and anti-HA antibodies. Scale bar $=10 \mu \mathrm{m}$

performed IF staining using Phalloidin (F-actin staining) and antibodies for GFP and HA on cells that were transfected with MTSS1-GFP and SCAMP1-HA. Increased cell-cell contacts that are shown by F-actin staining, were observed in SkBr3 and MDA-MB-453 cells expressing MTSS1 or SCAMP1 compared to cells expressing the empty vector (Fig. 6a, b). This increase was significantly important when MTSS1 and SCAMP1 were co-expressed (Fig. 6a, b). To determine whether this event is associated with MTSS1-mediated activation of RAC1-GTP, we used a Glutathione resin-based IP system to pull down active RAC1 (RAC-GTP) from whole-cell extracts that were obtained from $\mathrm{SkBr} 3$ cells transfected with the empty vector, MTSS1-GFP, SCAMP1-HA or a combination of MTSS1-GFP and SCAMP1-HA. The immunoprecipitates were analysed by IB using an antibody for RAC1. MTSS1expressing cells showed increased levels of RAC1-GTP expression as previously reported (Fig. 6c) ${ }^{7}$. SCAMP1expressing cells also showed an increased activity of RAC1GTP, similar to the one observed with MTSS1-expressing cells. However, co-expression of MTSS1 and SCAMP1 resulted in higher expression level of RAC1-GTP when compared to singularly expressing MTSS1 or SCAMP1 cells. The increased cell-cell contacts in the presence of MTSS1 and SCAMP1 and that were observed by F-actin staining suggests potential increase of cell-cell adhesions. To determine this, we performed a cell adhesion assay using the Vybrant ${ }^{\mathrm{Tm}}$ Cell Adhesion Assay. Indeed, significant increase in cell-cell adhesions were observed in the presence of MTSS1 and SCAMP1, and when compared to MTSS1 or SCAMP1 alone, and to the control (Fig. 6d, e). Although an increase of cell-cell adhesions were observed in the presence of either MTSS1 or SCAMP1, this increase was significantly lower than the one observed in the presence of expression of both molecules. Taken together, these observations highlight the cooperation of MTSS1 and Scamp1 in preventing HER2+/ER-/PR - cancer progression and provide further insights on early events associated with the pathogenesis of cancer invasion.

\section{Discussion}

Breast cancer is a heterogeneous disease resulting from a highly complex and diverse genomic portrait. Clinical management is determined by clinicopathology assessment, testing for hormonal (ER/PR) receptors and amplification of HER2. Essentially, this results in patient molecular classification that guides treatment regimen selection and survival risk. If improvement in individualised treatment is to be achieved, it is important to gain a better understanding of the cell signalling pathways that govern, or frustrate, patient molecular class membership. Risk of disease-specific death is increased by metastasis development and treatment resistance; the latter may result from interacting or opposing cell signalling pathways, calling for a new combination therapy approach ${ }^{24}$, to reduce metastasis risk. Metastasis involves a multi-step biological process and is characterised by loss of cell:cell adhesion, allowing cancer cells to invade through surrounding tissue ${ }^{25}$.

Cell-cell adhesions are maintained by functional units of multi-protein complexes that are organised into three general classes of proteins; the cell adhesion molecules/ adhesion receptors, the extracellular matrix (ECM) proteins, and the cytoplasmic plaque/peripheral membrane proteins ${ }^{26}$. These structures are essential for tissue organisation, homoeostasis and function. The maintenance and regulation of this natural and exceptional machinery rely on a complex network of intracellular and extracellular signals and cellular pathways. During tumour progression, cell-cell adhesions are affected by cytoskeleton alterations, disassembly of cell-cell junctions and consequent changes in cell morphology ${ }^{27,28}$. This enables malignant cancer cells to migrate, invade and metastasis. MTSS1 belongs to the IMD-family (IRSp53 and MIM (Missing in metastasis) domain) that act as cytoskeletal scaffold proteins and regulate cytoskeletal dynamics through interaction with Rac1, actin and actin-associated proteins $^{5-7,19,29}$. MTSS1 is highly expressed in primary tumours and its loss has been found to correlate with metastasis and poor prognosis in different types of cancer $^{9,15-22}$. However, other studies have shown that MTSS1 expression may be associated with increased invasion and metastasis in subtypes of malignant melanoma, non-small cell lung (NSCLC) and breast cancer $^{30-32}$. These observations indicate differences in functionality for MTSS1 according to tumour specificity. Although MTSS1 was expressed in some HER2+ molecular subtypes, we observed its expression was markedly decreased in clinical tumour samples with the 


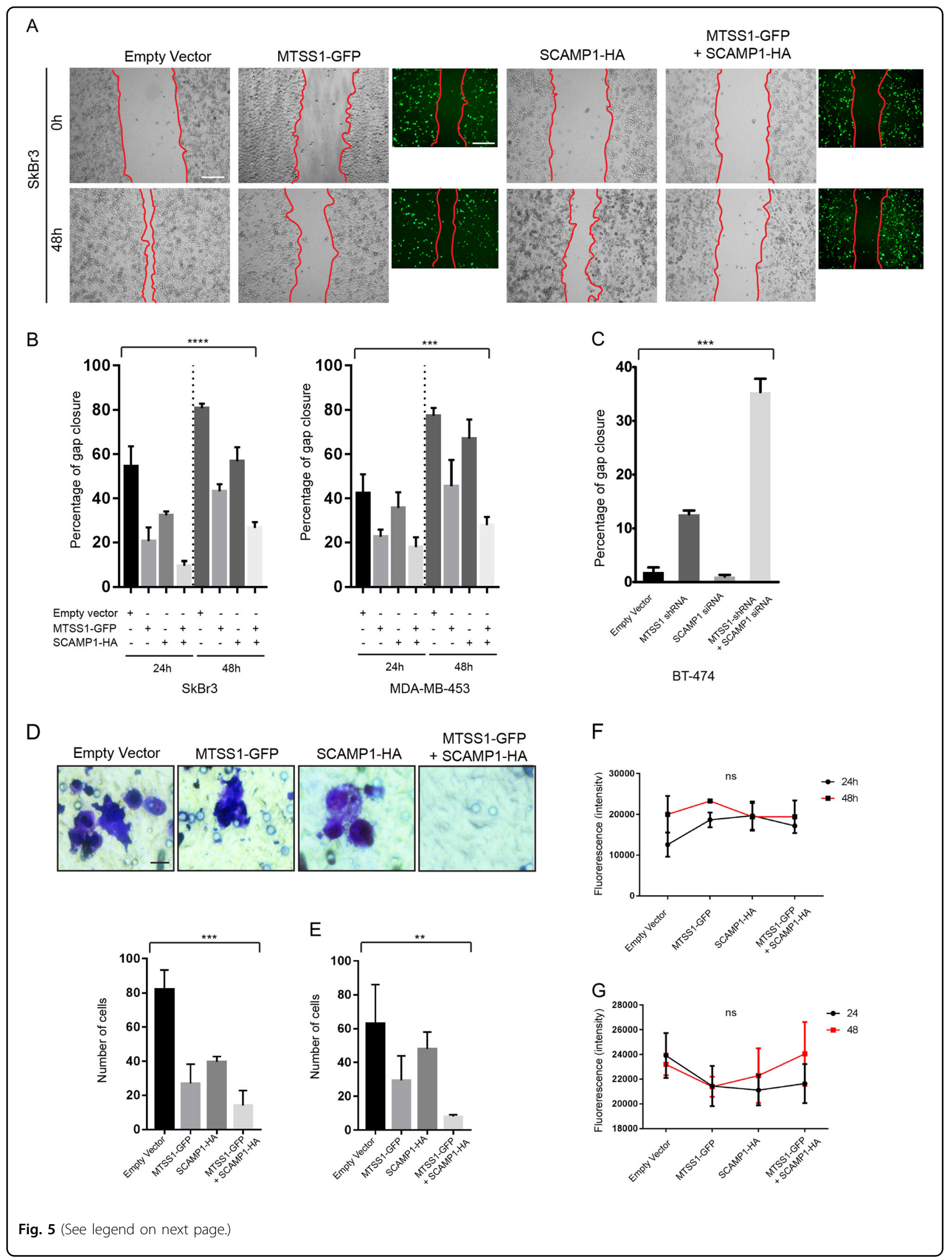


(see figure on previous page)

Fig. 5 MTSS1 and SCAMP1 expression prevents cell migration and invasion. $\mathbf{a}$, b Wound-healing assay (Scratch assay) using SkBr3 and MDAMB-453 expressing MTSS1-GFP, SCAMP1-HA or both constructs and the corresponding data quantifying gap closure at time points 0,24 and $48 \mathrm{~h}$ following scratching. ANOVA ${ }^{* * *} P=<0.0001$ and ${ }^{* *} P=0.0001$. Scale bar $=200 \mu \mathrm{m}$. c Wound-healing assay (Scratch assay) using BT-474 cells expressing empty vector, MTSS1-ShRNA, SCAMP1 siRNA or both and the corresponding data quantifying gap closure at time points $24 \mathrm{~h}$ following scratching. ANOVA ${ }^{* * *} P<0.0001$. d, e Well-cell invasion assay using SkBr3 and MDA-MB-453 cells expressing MTSS1-GFP, SCAMP1-HA or both constructs and the data relating to the number of invading cells after $48 \mathrm{~h}$ of culture. ANOVA ${ }^{* *} P=0.0001$ and ANOVA ${ }^{* *} P=0.0084$. Scale bar $=10$ $\mu \mathrm{m} . \mathbf{f}, \mathbf{g}$ Graphs representing measurements of cell proliferation of SkBr3 and MDA-MB-453 expressing MTSS1-GFP, SCAMP1-HA or both constructs (24 and $48 \mathrm{~h}$ post transfection). Two-way ANOVA $P(\mathrm{~ns})=0.1878$ ) and two-way ANOVA $P(\mathrm{~ns})=0.8040$

HER2+/ER-/PR - phenotype, suggesting hormonal androgen expression may mitigate for the loss of MTSS1 tumour suppressor functionality. This decrease of MTSS1 expression correlated with reduced survival and worse prognosis in HER2+/ER-/PR - breast cancer patients. Furthermore, this clinical finding was validated in our in vitro invasive models of breast cancer. We showed that restoring Mtss1 expression in HER2+/ER-/PR - breast cancer cell lines promoted cell-cell adhesion and prevented cell invasion. These events were associated with the capacity of MTSS1 to induce elevated levels of RAC1GTP, as previously reported ${ }^{7}$. Our clinical findings support a recent study showing decreased survival in trastuzumabtreated breast cancer patients with a HER2/ER - tumour profile compared to HER2/ER+ (HER2/luminal B) patients $^{33}$. The current findings provide a possible explanation for the difference seen in the two patient groups.

Although we have some understanding of the role of MTSS1 in cancer, cellular pathways that may regulate its function are not well-known. Using a system biology approach, we identified several potential interacting partners, of which SCAMP1 was selected for further studies. This selection was based on the role of this molecule in post-Golgi recycling pathways and in endosome cell membrane recycling ${ }^{10,11}$. MTSS1 transport to the cell surface may be facilitated by SCAMP1. Moreover, the role of SCAMP1 in cancer is still unknown. Indeed, we found that SCAMP1 expression correlated with Mtss1 expression in tumours with the HER $2+/ \mathrm{ER}-/ \mathrm{PR}-$ phenotype and its expression was decreased similarly to MTSS1 in the HER2 +/ER-/PR - breast cancer class. Likewise, restoring SCAMP1 expression in HER2+/ER-/PR - breast cancer cell lines also promoted cell-cell adhesion and prevented cell invasion. Importantly, co-expression of MTSS1 and SCAMP1 resulted in a more efficient inhibition of cell invasion and increased cell-cell adhesion in our cancer models when compared to the expression of Mtss1 or Scamp1 alone. Taken together, these results confirm our hypothesis of synergistic interaction between MTSS1mediated and SCAMP1-mediated cellular pathways in preventing HER2+/ER-/PR - breast cancer invasion. Our findings lead us to propose that further investigations are needed to assess the relationship between loss of MTSS1 in HER2 patients and its relationship to trastuzumab response.

\section{Materials and methods \\ Antibodies}

For this study, we used antibodies to MTSS1 (1:30 for IHC, LS-B1818, LifeSpan BioSciences, Seattle, WA, USA), MTSS1 (1:250 for IB, 4386, Cell Signaling Technology, Danvers, MA, USA), MTSS1 (1:50 for PLA, sc-101390, Santa Cruz Biotechnology, Dallas, TX, USA), SCAMP1 (1:25 for IHC, 1:100 for PLA, 15327-1-AP, Proteintech, Rosemont, IL, USA), SERPIN B13 (1:125 for IHC, NBP201312, Novus Biological, Littleton, CO 80120, USA), GFP (1:1000, ab1218, Abcam, Cambridge, UK), HA (1:1000 for IB, 1:200 for IF, A190-108 A, Bethyl Laboratories Inc., Montgomery, TX, USA), $\beta$-actin (1:5000 for IB, A5441, Sigma-Aldrich, St Louis, MO, USA).

\section{Plasmids and constructs}

MTSS1-GFP and SCAMP1-HA fusion genes were synthesised using custom Gene Synthesis Service (GenScript, Piscataway, NJ, USA) and incorporated into PUC57Kan cloning vector. The fusions genes PUC57Kan vector were further sub-cloned into PCDNA 3.1(+) expression vector (Invitrogen, ThermoFisher scientific) using $5^{\prime}$-Bam $\mathrm{H} 1$ and $3^{\prime}-X b a$ I restriction sites. MTSS1-shRNA lentiviral constructs were purchased from Sigma-Aldrich (SHCLNG-NM_014751) and were used as previously described $^{34}$. SCAMP1 siRNAs were purchased from QIAGEN (FlexiTube GeneSolution GS9522 for SCAMP1) and were used following manufacturer recommendations.

\section{Cell lines, growth conditions and cell transfection}

SkBr3 breast cancer cells (HTB-20, ATCC) were cultured in Lonza/12-168 McCoy's media with 10\% fetal bovine serum (FBS). MDA-MB-453 cells (ACC65, Leibniz Institute DSMZ-German Collection of Microorganisms and Cell Cultures) were cultured in 90\% Leibovitz's L-15 medium with $10 \%$ FBS. BT-474 cells (HTB-20, ATCC) were cultured in ATCC/46-XHBRI-CARE media with $10 \%$ FBS and $1.5 \mathrm{~g} /$ L Sodium Bicarbonate. Plasmids were transfected using Lipofectamine $^{\mathrm{TM}} 3000$ Reagent (L3000001, ThermoFisher Scientific) and following manufacturer recommendations.

\section{Cell migration, invasion, proliferation and adhesion assays}

For wound-healing assay (Scratch assay), SkBr3 and MDA-MB-453 cells expressing the empty vector, MTSS1- 


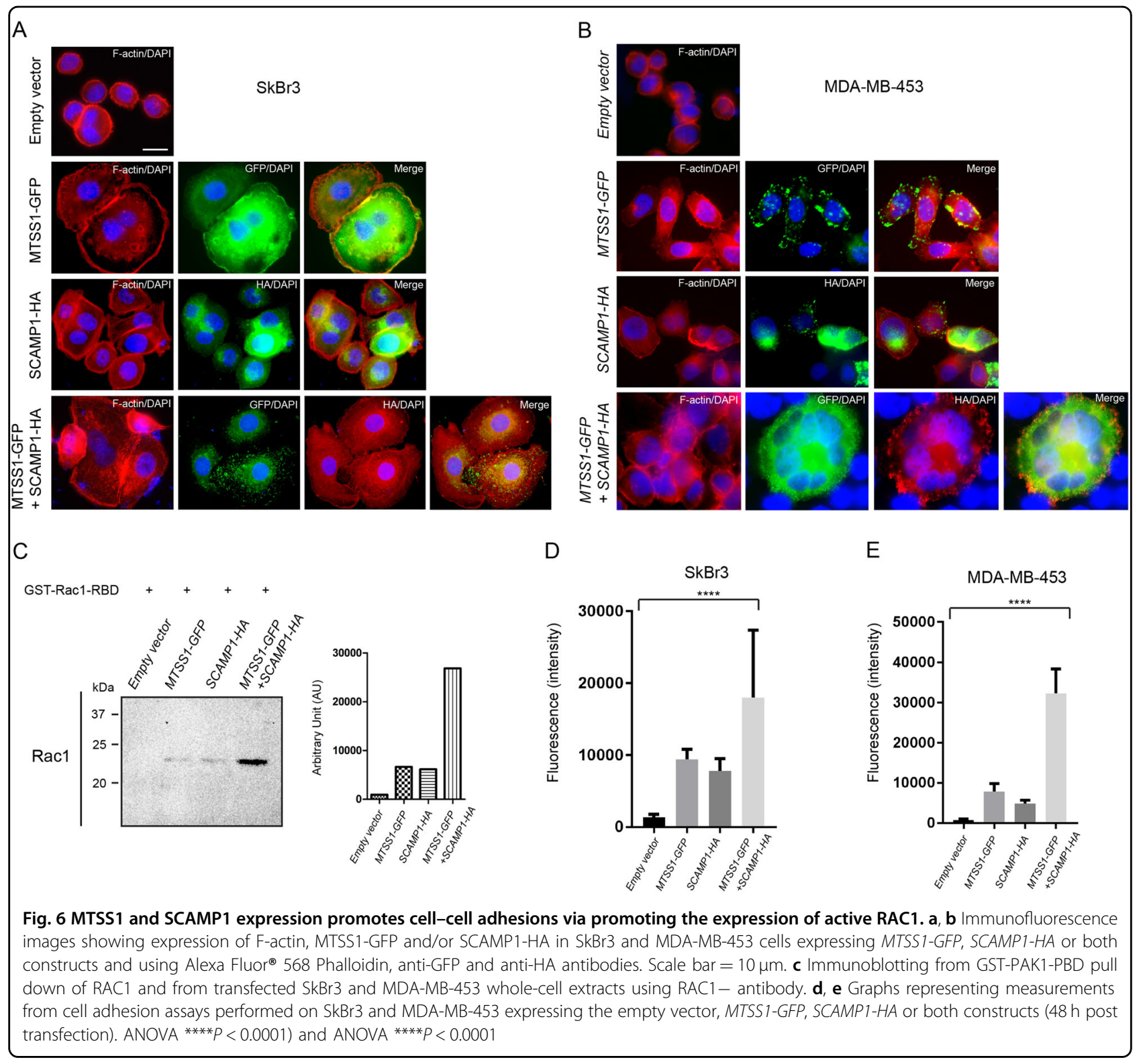

GFP, SCAMP1-HA or both were cultured to $80 \%$ confluence and serum starved for $24 \mathrm{~h}$, after which a scratch was made in the middle of each well using a $10 \mu$ pipette tip. Images from triplicate experiments at 0,24 and $48 \mathrm{~h}$ were taken and the distances between the edges of the scratch were measured at 3 different points using Carl Zeiss AxioVision software ${ }^{35}$ (Buczek et al., 2016). The measurements were expressed as percentages of gap closure. For the well-cell invasion assay, we used the colorimetric QCM ECMatrix Cell Invasion Assay (ECM550, Millipore, Ltd.) and the cell count of invasive cancer cells were performed following the manufacturer's recommendations. For the proliferation assay, cells were cultured (10000 cells per/well) and then transfected for $24 \mathrm{~h}$ and $48 \mathrm{~h}$. Media were removed and the proliferation was assessed using the CyQUANT ${ }^{\circledR}$ NF Cell Proliferation Assay Kit (C35007, Life Technologies, Ltd.) and following the manufacturer's recommendations. Cell-cell adhesions were assessed following transfections (48 h) and using Vybrant $^{\mathrm{Tm}}$ Cell Adhesion Assay Kit (V13181, Fisher Scientific), and the manufacturer's recommendations.

\section{IB and IP}

The experimental procedures were performed as described previously ${ }^{34}$. For IB, the cells lysed in $1 \times$ solution containing $50 \mathrm{mM}$ Tris- $\mathrm{HCl}(\mathrm{pH} 6.8), 100 \mathrm{mM}$ dithiothreitol, 2\% (w/v) SDS, 0.1\% (w/v) bromophenol blue, and $10 \%$ (v/v) glycerol, and loaded onto Tris/glycine SDS-polyacrylamide gels for electrophoresis. The proteins were transferred onto Amersham Hybond-P PVDF 
membranes (GE Healthcare). Membranes were blocked with $10 \%(\mathrm{w} / \mathrm{v})$ Marvel milk powder/phosphate-buffered saline (PBS) solution with $0.1 \%(\mathrm{v} / \mathrm{v})$ Tween-20 (PBST). Membranes were washed in PBST, membranes were incubated with primary antibodies (in blocking solution) overnight at $4{ }^{\circ} \mathrm{C}$ followed by washing and incubation with secondary antibodies for $1 \mathrm{~h}$ at room temperature prior to visualisation using Clarity ${ }^{\text {Tu }}$ Western ECL Substrate (BioRad Laboratories) and a CCD camera (SynGene). For IP, the cells were lysed in IP buffer (0.05 M Tris pH 7.4, $0.15 \mathrm{M}$ $\mathrm{NaCl}, 0.5 \%(\mathrm{v} / \mathrm{v})$ Triton X-100, and $0.001 \mathrm{M}$ EDTA). The extracts were pre-cleared for $1 \mathrm{~h}$ at $4{ }^{\circ} \mathrm{C}$ using Protein G Plus-Agarose beads (IP08, Calbiochem) and corresponding IgG. GFP antibody was incubated with agarose beads in IP buffer for $2 \mathrm{~h}$ before adding the pre-cleared extracts for overnight IP. The beads were washed 4 times in IP buffer and suspended in lysis buffer, after which samples were analysed by IB. For active Rac1 detection, we used the Active Rac1 Detection Kit and following manufacturer recommendations (\#8815, Cell Signaling Technology).

\section{Bioinformatics analysis}

\section{Monte Carlo Cross validation}

Prior to ANN training, the data was randomly divided into three subsets; $60 \%$ for training, $20 \%$ for testing (to assess model performance during the training process) and $20 \%$ for validation (to independently test the model on data completely blind to the model). This process of random sample cross validation also contributed to the reduction of over fitting to the data.

\section{Architecture}

The ANN modelling undertaken used a supervised learning approach applied to a three-layer multi-layer perceptron architecture. The initial weights matrix was randomised with a standard deviation of 0.1 to reduce the risk of over fitting the data. The ANN architecture was initially constrained to two hidden nodes in the hidden layer also for this reason. Hidden nodes and the output node incorporated a sigmoidal transfer function. During training, weights were updated by a feed-forward back propagation algorithm ${ }^{36}$. Learning rate and momentum were set at 0.1 and 0.5 , respectively. The output node was coded as 0 if the patient showed low expression of RAS, and 1 if the patient showed high expression of RAS.

\section{Network inference analysis}

Network inference analysis was undertaken on the top 100 probes from step 1 of the classifier development. Thus the single non-orthogonal probes were analysed. This approach $^{13,14}$ uses a suite of ANN models to study the inter-relationship between markers in a defined set. In the algorithm all markers are used to predict 1 marker and the weights of the model analysed. This is then repeated for each marker in turn so that in the process all markers are used as outputs and a matrix of interactions identified. This matrix is then filtered to identify the strongest interactions and the most connected/influential genes. The resulting interactions were presented using Cytoscape.

\section{Immunohistochemistry}

The relationship between MTSS1 to its carrier molecule, SCAMP1, and its stabilisation protein, SERPIN B13 was investigated in a cohort of wax embedded tissue microarrayed (TMA) breast cancer using immunohistochemistry. The TMA comprises tumour samples obtained from unselected patients diagnosed with primary operable breast cancer (TNM stage I-IIIa) that presented at the Nottingham Breast Unit between 1988 and 1998. Prospectively collected clinical and pathology data is available including development of recurrence and distant metastases (DM), survival time and disease-free interval (DFI). Breast cancer-specific survival (BCSS) was defined as the time (in months) from the date of the primary surgical treatment to the time of death from breast cancer. DFI was defined as the interval (in months) from the date of the primary surgical treatment to the first locoregional or distant metastasis. Patient management and treatment regimens have been previously described $^{1}$. Protein expression was immunohistochemically assessed using a Ventana Benchmark Ultra platform (Roche Group, Arizona, US). Malignant epithelium was microscopically dichotomously scored for levels of staining intensity where 0 : negative or low intensity staining, and 1 : moderate to strong intensity. REMARK guidelines ${ }^{37}$ were used in the whole patient series and the number of scorable patients was found to be less than those originally arrayed due to tissue detachment and absence of malignant tissue. A Pearson's $\chi^{2}$-test with cross-tabulation tables was performed to assess associations between the biomarkers and clinical survival data, using SPSS (Version 21; IBM, US). Kaplan-Meier plots with log-rank tests were used to model biomarker associations for disease-specific survival (DSS) and time to metastasis development. $\chi^{2}$-tests were performed on the whole (unselected) patient cohort, followed by subset analysis of HER2 (cerbB2)-positive patients, and Pearson's correlation tests were used to assess the association between different antigen biomarkers with clinical outcome. HER2+ patients were stratified according to hormonal oestrogen and progesterone receptor status.

\section{IF staining and PLA}

The IF staining was performed as previously described $^{34}$. Cells were fixed in $4 \%(\mathrm{w} / \mathrm{v})$ paraformaldehyde and then treated as follows: the sections or cancer cells were washed three times in $1 \times$ PBS for 10 min each, blocked and permeabilised in $10 \%(\mathrm{w} / \mathrm{v})$ bovine serum albumin in $0.1 \%(\mathrm{v} / \mathrm{v})$ PBS-Tween, incubated overnight with primary antibody (in blocking solution), washed three times for 10 
min each with $1 \times$ PBS, incubated for $1 \mathrm{~h}$ with secondary antibody (in blocking solution) and washed three times with $1 \times$ PBS. Sections and melanoma cells were counterstained and mounted with DAPI fluorescent medium (Vector Laboratories) for IF microscopy. The PLA assay was performed using the Duolink ${ }^{\oplus}$ In Situ Red Starter Kit Mouse/Rabbit (DUO92101, Sigma-Aldrich, St Louis, MO, USA) and following the manufacturer recommendations. The number of dots/cell was determined by counting the number of dots from three independent images.

\section{Acknowledgements}

This work was supported by the John and Lucille van Geest Foundation, and the John van Geest Cancer Research Centre, Nottingham Trent University. This study was supported by the European Union's Horizon 2020 research and innovation program under the Marie Sklodowska-Curie grant agreement no 641549, Immutrain.

\section{Author details}

The John van Geest Cancer Research Centre, School of Science and Technology, Nottingham Trent University, Nottingham NG11 8NS, UK. ${ }^{2}$ Department of Cellular Pathology, Queen's Medical Centre, Nottingham University Hospitals Trust, Nottingham NG7 2UH, UK. ${ }^{3}$ Department of Biology, College of science for women, University of Baghdad, Baghdad, Iraq

\section{Conflict of interest}

The authors declare that they have no conflict of interest.

\section{Publisher's note}

Springer Nature remains neutral with regard to jurisdictional claims in published maps and institutional affiliations.

Supplementary Information accompanies this paper at https://doi.org/ 10.1038/s41419-018-0364-9.

Received: 7 August 2017 Revised: 10 January 2018 Accepted: 30 January 2018

Published online: 01 March 2018

\section{References}

1. Jiang W. G. et al. Tissue invasion and metastasis: Molecular, biological and clinical perspectives. Semin. Cancer Biol. 35, (2015).

2. Fife, C. M., McCarroll, J. A. \& Kavallaris, M. Movers and shakers: cell cytoskeleton in cancer metastasis. Br. J. Pharmacol. 171, 5507-5523 (2014).

3. Sundaresan, S., Penuel, E. \& Sliwkowski, M. X. The biology of human epidermal growth factor receptor 2. Curr. Oncol. Rep. 1, 16-22 (1999).

4. Alvarez, R. H., Valero, V. \& Hortobagyi, G. N. Emerging targeted therapies for breast cancer. J. Clin. Oncol. 28, 3366-3379 (2010).

5. Machesky, L. M. \& Johnston, S. A. MIM: a multifunctional scaffold protein. J. Mol. Med. 85, 569-576 (2007).

6. Saarikangas, J. et al. Missing-in-metastasis MIM/MTSS1 promotes actin assembly at intercellular junctions and is required for integrity of kidney epithelia. J. Cell Sci. 124, 1245-1255 (2014).

7. Dawson, J. C., Bruche, S., Spence, H. J., Braga, V. M. \& Machesky, L. M. Mtss1 promotes cell-cell junction assembly and stability through the small GTPase Rac1. PLoS ONE 7, e31141 (2012).

8. Hicks, D. G. et al. Loss of breast cancer metastasis suppressor 1 protein expression predicts reduced disease-free survival in subsets of breast cancer patients. Clin. Cancer Res. 12, 6702-6708 (2006).

9. Parr, C. \& Jiang, W. G. Metastasis suppressor 1 (MTSS1) demonstrates prognostic value and anti-metastatic properties in breast cancer. Eur. J. Cancer 45, 1673-1683 (2009).

10. Castle, A. \& Castle, D. Ubiquitously expressed secretory carrier membrane proteins (SCAMPs) 1-4 mark different pathways and exhibit limited constitutive trafficking to and from the cell surface. J. Cell Sci. 118, 3769-3780 (2005).

11. Law, A. H. Y., Chow, C.-M. \& Jiang, L. Secretory carrier membrane proteins. Protoplasma 249, 269-283 (2012).

12. Goldenring, J. R. A central role for vesicle trafficking in epithelial neoplasia: intracellular highways to carcinogenesis. Nat. Rev. Cancer 13, 813-820 (2013).

13. Tong, D. L., Boocock, D. J., Dhondalay, G. K. R., Lemetre, C. \& Ball, G. R. Artificial neural network inference (ANNI): a study on gene-gene interaction for biomarkers in childhood sarcomas. PLoS ONE 9, e102483 (2014).

14. Abdel-Fatah, T. M. et al. SPAG5 as a prognostic biomarker and chemotherapy sensitivity predictor in breast cancer: a retrospective, integrated genomic, transcriptomic, and protein analysis. Lancet Oncol. 17, 1004-1018 (2016).

15. Parr, C. \& Jiang, W. G. Metastasis suppressor 1 (MTSS1) demonstrates prognostic value and anti-metastatic properties in breast cancer. Eur. J. Cancer 45, 1673-1683 (2009)

16. Zeleniak, A. E., Huang, W., Brinkman, M. K., Fishel, M. L. \& Hill, R. Loss of MTSS1 results in increased metastatic potential in pancreatic cancer. Oncotarget $\mathbf{8}$, 16473 (2017).

17. Schemionek, M. et al. Mtss1 is a critical epigenetically regulated tumor suppressor in CML. Leukemia 30, 823-830 (2016).

18. Zhang, S. \& Qi, Q. MTSS1 suppresses cell migration and invasion by targeting CTTN in glioblastoma. J. Neurooncol. 121, 425-431 (2015).

19. Xie F., Ye L., Ta M., Zhang L., Jiang W. G. MTSS1: a multifunctional protein and its role in cancer invasion and metastasis. Front. Biosci. 2011; 3: 621-631.

20. Hofer, M. D. et al. The role of metastasis-associated protein 1 in prostate cancer progression. Cancer Res. 64, 825-829 (2004).

21. Kayser, G. et al. Downregulation of MTSS1 expression is an independent prognosticator in squamous cell carcinoma of the lung. Br. J. Cancer 112, 866 (2015).

22. Mohamed, M. M. \& Sloane, B. F. Cysteine cathepsins: multifunctional enzymes in cancer. Nat. Rev. Cancer 6, 764 (2006).

23. Higgins, W. J. et al. Heparin enhances serpin inhibition of the cysteine protease cathepsin L. J. Biol. Chem. 285, 3722-3729 (2010).

24. Mayer, I. A. et al. A phase Ib study of alpelisib (BYL719), a PI3Ka-specific inhibitor, with letrozole in ER+/HER2- metastatic breast cancer. Clin. Cancer Res. 23, 26-34 (2017).

25. McAllister, S. S. \& Weinberg, R. A. The tumour-induced systemic environment as a critical regulator of cancer progression and metastasis. Nat. Cell Biol. 16, 717 (2014).

26. Gumbiner, B. M. Cell adhesion: the molecular basis of tissue architecture and morphogenesis. Cell 84, 345-357 (1996).

27. Alexandrova, A. Y. Plasticity of tumor cell migration: acquisition of new properties or return to the past?. Biochemistry (Mosc.) 79, 947-963 (2014).

28. Brooke, M. A., Nitoiu, D. \& Kelsell, D. P. Cell-cell connectivity: desmosomes and disease. J. Pathol. 226, 158-171 (2012).

29. Loberg, R. D. et al. Differential expression analysis of MIM (MTSS1) splice variants and a functional role of MIM in prostate cancer cell biology. Int. J. Oncol. 26, 1699-1705 (2005).

30. Mertz, K. D. et al. MTSS1 is a metastasis driver in a subset of human melanomas. Nat. Commun. 5, 3465 (2014).

31. Giacobbe, A. et al. p63 controls cell migration and invasion by transcriptional regulation of MTSS1. Oncogene 35, 1602-1608 (2016).

32. Ling, D. J. et al. Differential effects of MTSS1 on invasion and proliferation in subtypes of non-small cell lung cancer cells. Exp. Ther. Med. 12, 1225-1231 (2016).

33. McGuire A. et al. Differential impact of hormone receptor status on survival and recurrence for HER2 receptor-positive breast cancers treated with Trastuzumab. Breast Cancer Res. Treat. 1-9 (2017).

34. Mathieu, M. G. et al. The helicase HAGE prevents interferon-a-induced PML expression in $\mathrm{ABCB} 5+$ malignant melanoma-initiating cells by promoting the expression of SOCS1. Cell Death Dis. 5, e1061 (2014).

35. Buczek, M. E. et al. Cytoplasmic PML promotes TGF- $\beta$-associated epithelial-mesenchymal transition and invasion in prostate cancer. Oncogene 35, 3465 (2016).

36. Lancashire, L., Sutton, A., Rees, R. \& Ball, G. O-33 An artificial neural network based algorithm for predicting continuous time to event data in breast cancer. Eur. J. Cancer Suppl. 8, 12-13 (2010).

37. McShane, L. M. et al. REporting recommendations for tumour MARKer prognostic studies (REMARK). Eur. J. Cancer 41, 1690-1696 (2005). 